\title{
Multi-Length Scale X-ray Tomography of Li-ion Batteries
}

\author{
P.R. Shearing*, R. Bradley**, J. Gelb***, N.P. Brandon*, P.J. Withers** \\ * Dept. of Earth Science and Engineering, Imperial College London, UK \\ ** Henry Moseley X-ray Imaging Facility, School of Materials, University of Manchester, \\ $\mathrm{UK}$ \\ *** Xradia Inc, Pleasanton, CA
}

Li-ion batteries commonly consist of two porous electrodes divided by a porous separation layer. As is typical of functional materials, there is a direct link between the microstructure of these electrodes and the performance of the cell, but the widely varying length scales make characterization a challenging task. Microstructure is known to affect a broad range of physical phenomena and is intimately linked with capacity, conductivity, charge transfer, and failure. These phenomena in Li-ion batteries occur over a range of length scales, from the formation of SEI layers occurring at the nanometer length scale to the catastrophic failure of battery architectures visible by the naked eye.

In spite of the importance of microstructure in dictating battery performance, the 3D structures of these materials are not fully understood. Modern developments in the field of $\mathrm{x}$-ray computed microtomography and nanotomography (micro-CT and nano-CT), however, are providing new insight into the highly complex porous geometries that are characteristic of Li-ion battery electrodes [1-2]. The uniquely non-destructive nature of this technique makes it possible to repeatedly study Li-ion battery electrodes in 3D at multiple length scales. This, further, opens the door to characterization of microstructural evolution processes associated with cell performance and degradation, which may provide the critical information for understanding operational and failure mechanisms.

Here, we present multi-length scale tomography of materials for Li-ion batteries, encompassing the range from individual electrode particles to full commercial batteries. Utilizing laboratory and synchrotron imaging in tandem, we demonstrate a cross-platform capability for mapping these composite materials for structure, composition, and operational properties. We explore the relationship between microstructural information obtained at these length scales and challenge some assumptions of microstructural homogeneity extant in many models of battery performance and failure.

\section{References}

[1] P. R. Shearing et al, Electrochem. Comm. 12 (2010) 374-377.

[2] A. Tkachuk et al, Z. Krystallogr. 222 (2007) 650-655. 


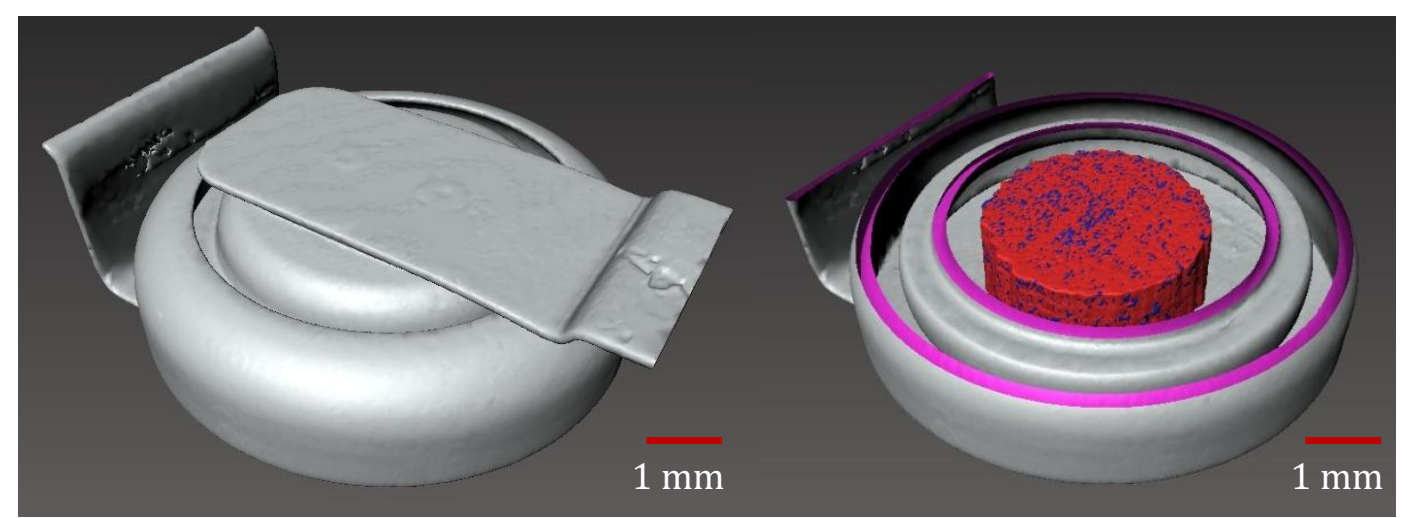

FIG. 1. 3D rendering of a commercial Li-ion coin cell battery as imaged by $\mathrm{x}$-ray microtomography. Analysis of the electrode (right) enables precise quantification of the different material phases.

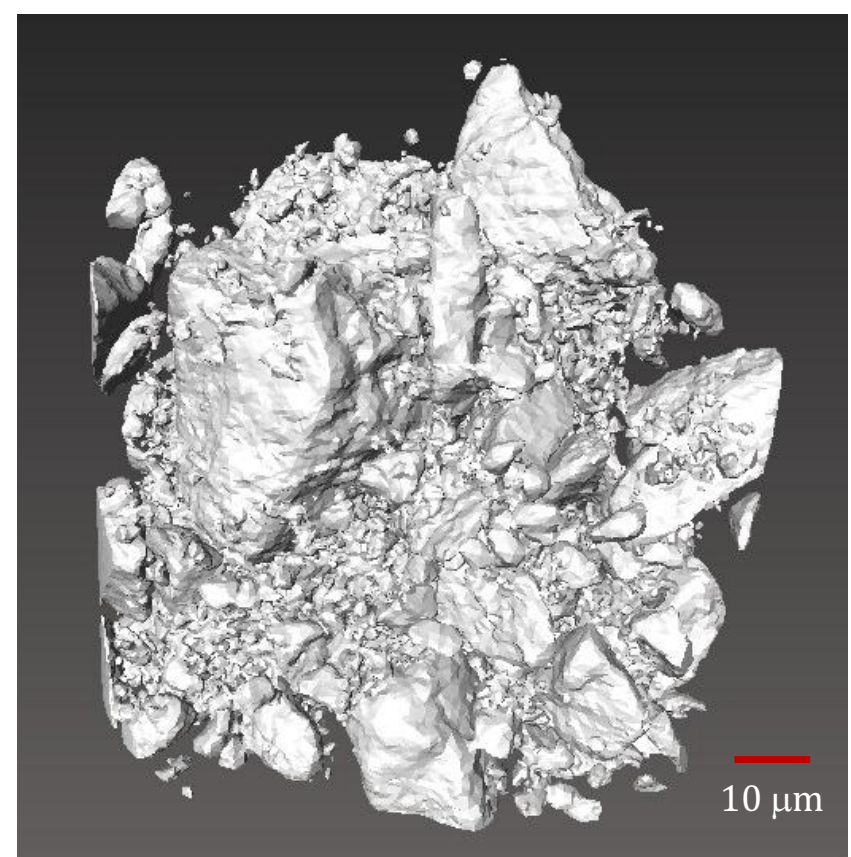

FIG. 2. 3D rendering of a porous cathode as imaged by laboratory x-ray nanotomography. From these results, quantifications of important microstructure parameters, such as volume fraction analysis and surface area of individual particles, are immediately available. 